\title{
PAPER
}

\section{Position detection of small objects in indoor environments using coded acoustic signal}

\author{
Kuramitsu Nishihara ${ }^{1}$, Tadashi Yamaguchi ${ }^{2, *}$ and Hiroyuki Hachiya ${ }^{3, \dagger}$ \\ ${ }^{1}$ Graduate School of Science and Technology, Chiba University \\ ${ }^{2}$ Graduate School of Advanced Integration Science, Chiba University \\ ${ }^{3}$ Graduate School of Science and Engineering, Tokyo Institute of Technology
}

(Received 2 March 2007, Accepted for publication 31 July 2007)

\begin{abstract}
An acoustic method that can be used in air has the potential to allow for a fast and accurate characterization of objects in air. Nevertheless, it is difficult to identify acoustic signals from small objects clearly because of environmental noise and the scattering of sound on the object surface. Therefore, a sensing system that enables the measurement of small objects in air must be developed. In this study, we performed the localization of small objects of size comparable to the sound wavelength using an M-sequence signal and the phase information of received signals in a noisy indoor environment. Using the M-sequence signal, we are able to improve the SN ratio and to measure in a stable manner the reflected waves that cannot be detected using a conventional impulse. The arrival direction information was used to extract signals reflected by targets from unwanted signals of the floor or ceiling. Using an M-sequence signal and the arrival direction information, the position detection of small objects in the indoor environment was enabled.
\end{abstract}

Keywords: Acoustic sensing, M-sequence signal, Arrival direction, Acoustic imaging

PACS number: 43.28.Tc, 43.30.Vh, 43.60.Lq [doi:10.1250/ast.29.15]

\section{INTRODUCTION}

Many studies on acoustic sensing in air have been reported as an effective method of acquiring information about an object such as its distance, shape and surface configuration, and environmental information such as temperature or wind velocity [1-8]. Nevertheless, it is difficult to identify acoustic signals of small objects clearly in indoor environments because of strong multiple reflections on the wall, the scattering of signals on the object surface, and background noise. A sensing system that enables indoor measurement must be developed. In particular, it is important to improve the $\mathrm{SN}$ ratio in the measurement system because it is one of the main factors determining the accuracy of sensing.

In this study, we performed the position detection of small objects of size comparable to the sound wavelength using an $\mathrm{M}$-sequence signal in a noisy indoor environment. Using the M-sequence signal, we were able to improve the SN ratio and measure in a stable manner the reflected waves that cannot be detected using a conventional impulse. In addition, we tried to extract signals reflected

*e-mail: yamaguchi@faculty.chiba-u.jp

†e-mail: hachiya@ctrl.titech.ac.jp by targets from unwanted signals of the floor or ceiling using the arrival direction of the received signals, which was obtained from the phase information of multiple microphones.

\section{METHOD}

\subsection{M-sequence Signal}

An M-sequence signal is a burst signal phase-modulated by a periodic pseudorandom binary control signal (0 or 1) known as an M-sequence. This signal is often used in the field of communication, measurement and system control. In an underwater acoustic measurement, it has been applied to ocean acoustic tomography $[9,10]$.

The M-sequence of degree $n$ is made using a circuit operation consisting of a shift register with $n$ stages and XOR (Exclusive or, $\oplus$ ) gates. Figure 1 shows a circuit that generates the M-sequence. Its sequence length $N$ is given by $N=2^{n}-1$ when the coefficients $f_{i}$ are chosen suitably. The sequence whose length is $2^{n}-1$ is the M-sequence $b_{k}(k=0,1,2, \cdots, N-1)$. The circuit is represented as a characteristic polynomial and given by

$$
f(x)=\sum_{j=0}^{n} f_{j} x^{j} \quad\left(f_{0}, f_{n}=1\right) .
$$




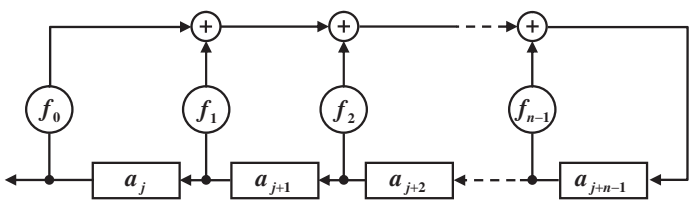

Fig. 1 Circuit for generating M-sequence of degree $n$.

Using this sequence, the M-sequence signal of degree $n$ is given by

$$
\begin{gathered}
s(t)=A \sin \left(\omega t+\phi_{k}(t)\right) \\
\phi_{k}(t)= \begin{cases}0 & \left(b_{k}=1\right) \\
\pi & \left(b_{k}=0\right)\end{cases} \\
k \frac{T}{N} \leq t<(k+1) \frac{T}{N} \quad(k=0,1,2, \cdots, N-1),
\end{gathered}
$$

where $A$ is the amplitude of the carrier signal, $\omega$ is the angular frequency, $b_{k}$ is the value of the $\mathrm{M}$-sequence and $T$ is the period of the signal. When this signal is correlated with a discrete $\mathrm{M}$-sequence, the result is given by

$$
\begin{gathered}
p(t)=C(t) \sin \omega t \\
C(t)= \begin{cases}N & (l T \leq t<T / N+l T) \\
-1 & \text { other } \quad(l=0,1,2, \cdots),\end{cases}
\end{gathered}
$$

where $l$ is the number of sequence periods. By discrete cross correlation of the signal, it is possible to achieve a high resolution that is equivalent to the resolution when a pulse wave in a digit of the M-sequence is transmitted and the $\mathrm{SN}$ ratio is improved due to the processing gain.

Figure 2 shows an example of the $\mathrm{M}$-sequence signal of degree 3 and a processing result. The high correlation value that maintains information about the carrier signal appears only in the first digit of the sequence. The precise measurement is enabled because it is able to acquire the phase information of the signal.

\subsection{Experiment}

Figure 3 shows a schematic diagram of the measurement system. In this measurement, the phase modulated $\mathrm{M}$-sequence signal, centered at $25 \mathrm{kHz}$, is transmitted from

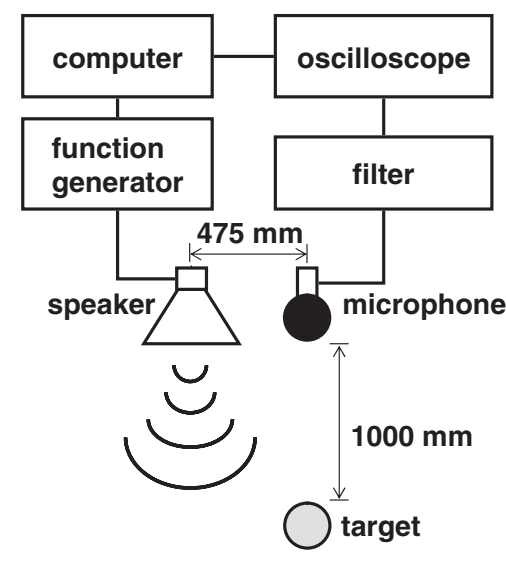

Fig. 3 Measurement system.

a card speaker (Panasonic, WM-R57A). The wavelength is about $14 \mathrm{~mm}$. The degree of the $\mathrm{M}$-sequence is 10 and there are 3 cycles per digit. This transmitting frequency was chosen by considering the acoustic attenuation in the medium, the spatial resolution and the card speaker characteristics [11]. The sequence is repeated 3 times during each transmission to eliminate the effect of a sequence truncation. It is theoretically predicted that the SN ratio is improved by $30.1 \mathrm{~dB}$ using the M-sequence of degree 10 . The characteristics of the transmitted signal are summarized as follows:

a) carrier frequency $f_{0}=25 \mathrm{kHz}$,

b) digit length $=3$ cycles of $25 \mathrm{kHz}$ carrier $=0.12 \mathrm{~ms}$,

c) sequence length $N=1,023$ digits $(122.76 \mathrm{~ms})$, and

d) transmission length $=3$, sequence period $=368.28 \mathrm{~ms}$.

The signal received by microphones (B\&K, Type4939) is amplified and filtered by a high-pass filter with a cutoff frequency of $10 \mathrm{kHz}$, then digitized at a sampling rate of $250 \mathrm{kHz}$. Figure 4 shows the configuration of an experiment in an indoor space. The targets were 5 stainless steel pipes with diameters of $13 \mathrm{~mm}$, which were of size comparable to the sound wavelength, and they were located at distances between 1 and $2 \mathrm{~m}$ from the microphone and positioned in the measurement space shown in Fig. 4.

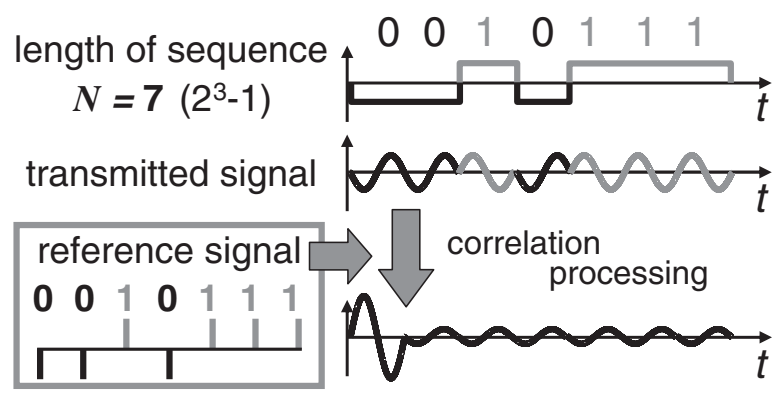

Fig. 2 M-sequence signal of degree 3.

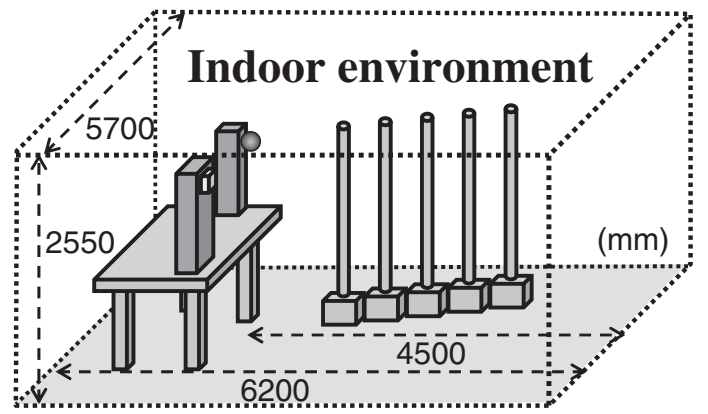

Fig. 4 Configuration of objects. 
The speaker and the microphone were located at a height of $1,200 \mathrm{~mm}$ and pipes were $1,840 \mathrm{~mm}$ high. All experiments were performed under stable room temperature. The measurement of distance is calculated using the speed of sound and the travel time. An approximate value of the speed of sound, $c(\mathrm{~m} / \mathrm{s})$, can be calculated from

$$
c=331.5+0.6 \times T_{\mathrm{c}},
$$

where $T_{\mathrm{c}}$ is the temperature in ${ }^{\circ} \mathrm{C}$.

\subsection{Acoustic Imaging}

For the accuracy validation, we formed the image of a circumference space by applying a simple acoustic imaging method for impulsive sound [12]. At each coordinate position $(x, y)$ on the image, the amplitude values of each channel at a corresponding travel time are arranged and accumulated. The travel time is given by

$$
t=\frac{\sqrt{\left(x_{\mathrm{s}}-x\right)^{2}+y^{2}}+\sqrt{\left(x_{\mathrm{r}_{i}}-x\right)^{2}+y^{2}}}{c},
$$

where $x_{\mathrm{s}}$ is the source position and $x_{r_{i}}$ is the position of each receiver. $c$ is the speed of sound, that was estimated from the measured temperature. To form the image, we captured reflected signals while moving the microphone horizontally every $10 \mathrm{~mm}$ from 0 to $300 \mathrm{~mm}$.

\section{RESULTS AND DISCUSSION}

\subsection{Comparison between M-Sequence Signal and Conventional Impulsive Signal}

To validate the effect of the M-sequence signal, we compared it with the received signals produced by transmitting the conventional impulsive signal. The received signal and the correlated signal of the M-sequence signal are shown in Fig. 5. Because the M-sequence signal of 3 sequence periods is transmitted, 3 similar signals appear in the correlated signal. Figure 6 shows the received signal produced by transmitting the conventional impulsive signal and a part of the correlated signal of the M-sequence signal in the second sequence period. For comparison, the

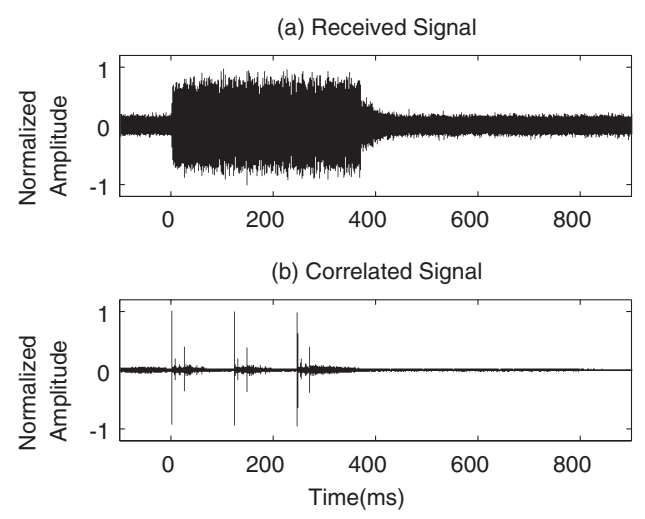

Fig. 5 Received signal and correlated signal of M-sequence.

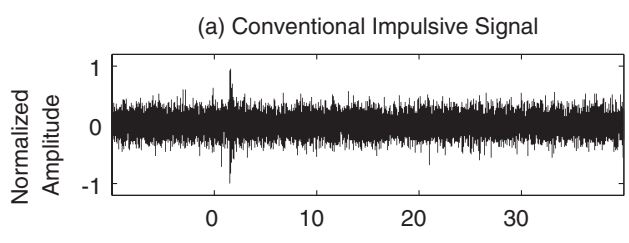

(b) Correlated Signal of M-sequence Signal

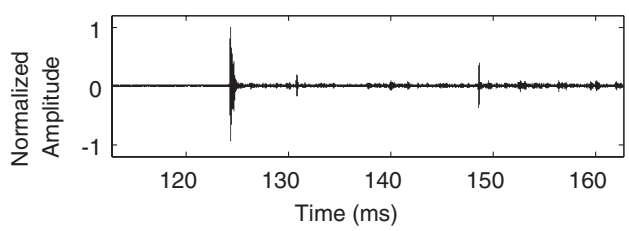

Fig. 6 Comparison of the conventional impulsive signal and the M-sequence signal.

conventional signal, centered at $25 \mathrm{kHz}$ and 3 cycles, is transmitted. The direct wave, which traveled from the speaker to the microphone directly, was received at $1.5 \mathrm{~ms}$ $(124.3 \mathrm{~ms})$ in both signals. On the other hand, it is confirmed that the various reflected signals which cannot be detected using the conventional impulsive signal can be detected by transmitting the $\mathrm{M}$-sequence signal. In addition, the SN ratio was improved by approximately $30 \mathrm{~dB}$ when the correlated signal of the M-sequence signal is compared with the conventional impulsive signal, where signal amplitude is estimated the effective value of the amplitude of the direct wave and noise level is estimated from the standard deviation of the amplitude of the signal during the period $20 \mathrm{~ms}$ before the direct wave is received. The noise was an acoustic background noise that contained broadband components. The measured $30 \mathrm{~dB}$ improvement of the SN ratio agrees well with the predicted value, $30.1 \mathrm{~dB}$, for the M-sequence of degree 10 .

In this result, it is thought that the measurement using the M-sequence signal is an effective method in such an environment.

\subsection{Estimation of the Arrival Directions}

We can estimate the arrival direction of the signals using the travel time difference and/or the phase difference of the signals received at two microphones. Figure 7 shows the configuration of receivers for the measurement of arrival direction. We can measure the arrival direction more precisely using the phase information. From two received signals $r_{i}(t)$ given by

$$
r_{i}(t)=A_{i}(t) \sin \left(\omega t+\phi_{i}(t)\right) \quad(i=1,2),
$$

we can get the demodulated complex signals $d_{i}(t)$

$$
d_{i}(t)=A_{i}(t) \exp \left(j \phi_{i}(t)\right) \quad(i=1,2),
$$

where $i$ is the number of microphones. When the phase difference of the two received signals $\Delta \phi(t)(\mathrm{rad})$ is given by 


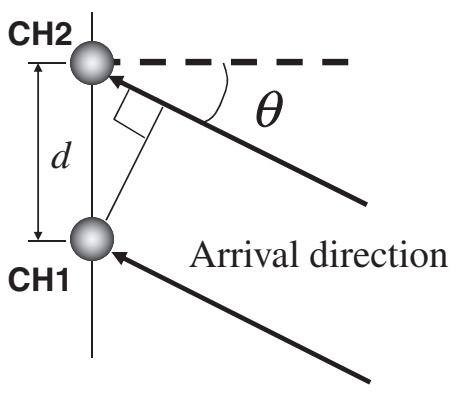

Fig. 7 Arrival direction of acoustic signals.

$$
\Delta \phi(t)=\phi_{1}(t)-\phi_{2}(t)=\arg \left(d_{1}(t) \times d_{2}(t)^{*}\right),
$$

the arrival direction $\theta(t)$ can be calculated using

$$
\theta(t)=\arcsin \frac{\lambda}{2 \pi d} \Delta \phi(t),
$$

where $\lambda$ is the wavelength and $d$ is the spacing of two microphones [13].

We performed the arrival direction measurement in the same configuration as that of Fig. 4. In this measurement, we used two microphones arranged in the vertical direction, and the distance between the two microphones was $7 \mathrm{~mm}$. The measurement results are shown in Fig. 8 . Signals reflected from a target in correlated M-sequence signals are shown in Figs. 8(a) and (b). The estimated arrival direction is shown in Fig. 8(c). A signal reflected from a target is recognized at approximately $128.8 \mathrm{~ms}$. The arrival direction of sound from a target is estimated to be about $0^{\circ}$, which means that sound comes from the horizontal direction. The M-sequence signal enables not only the detection of small objects but also the measurement of the arrival direction.

The arrival direction information is useful for extracting reflected signals of targets from unwanted signals of the floor or ceiling. The details will be described later.

\subsection{Acoustic Imaging Using M-Sequence Signal}

Figure 9 shows an acoustic image of targets produced

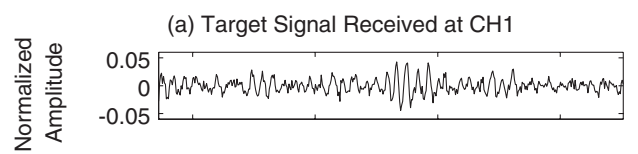

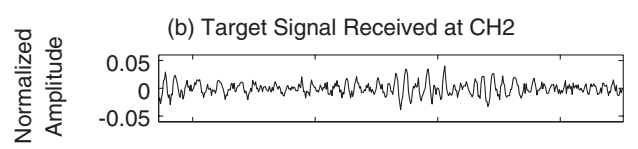

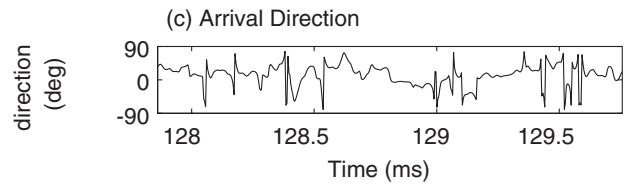

Fig. 8 Measurement result of the arrival direction.

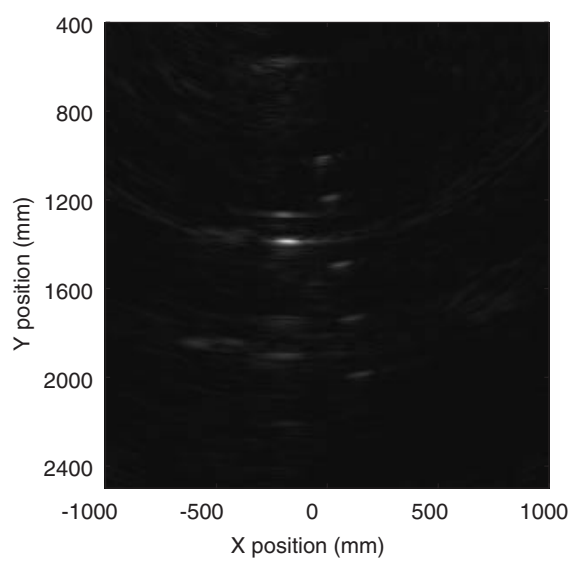

Fig. 9 Acoustic image produced by correlated signals.

using signals while moving a microphone horizontally every $10 \mathrm{~mm}$ from 0 to $300 \mathrm{~mm}$. The origin of the imaging area agrees with the initial position of the moving receiver. An acoustic image over an area of 2,000 $\mathrm{mm}(x=-1,000$ to $1,000 \mathrm{~mm}) \times 2,000 \mathrm{~mm} \quad(y=400$ to $2,400 \mathrm{~mm})$ was formed in this figure. This measurement configuration is equivalent to a one-dimensional array, in which the vertical directivity of each element is wide.

Figure 10 shows the configuration of five targets for Fig. 9. The five targets $t_{1}, t_{2}, t_{3}, t_{4}$ and $t_{5}$ were located at $(0,1,000),(50,1,200),(100,1,500),(150,1,750)$ and $(200$, $2,000)$, respectively. $t_{1}, t_{2}$ and $t_{3}$ were arranged at uneven intervals for accuracy verification. $t_{3}, t_{4}$ and $t_{5}$ were arranged at even intervals.

In the acoustic image (Fig. 9), the reflection from the ceiling and the floor at around $(-200,1,400)$ is also included as well as that from the five targets. The localization of small objects was realized using an Msequence signal. Because the reflection from the ceiling and the floor is strong, the image intensity of targets is relatively weak.

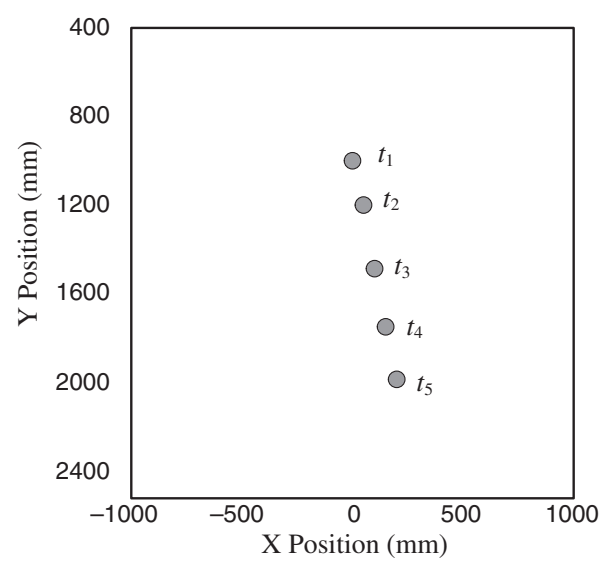

Fig. 10 Configuration of targets. 


\subsection{Imaging of the Selected Directional Response}

To remove signals from the ceiling and/or the floor in Fig. 9, a resolution power in the vertical direction is required. Imaging of the three-dimensional space can be performed using a two-dimensional array, but a system becomes complicated, and it becomes a problem in practical use. The signals from the ceiling and/or the floor arrive at the microphone from the vertical direction, which is different from the direction of the targets. We used the arrival angle in the vertical direction to remove the images of the ceiling and/or the floor.

It was confirmed that the arrival direction of the received signal can be acquired using two microphones; thus, we formed the acoustic image using two microphones placed in the vertical direction. The two microphones, which were configured vertically, were moved horizontally every $10 \mathrm{~mm}$ from 0 to $300 \mathrm{~mm}$. From a pair of correlated results of two received M-sequence signals, we can calculate the arrival direction of reflected sound as shown in Fig. 8.

Figures 11-13 show the images constructed by considering the vertical arrival direction. Figure 11 shows the generated image using signals that come from $-30^{\circ}$ to $30^{\circ}$ with respect to the vertical direction. The signals from the targets are emphasized by suppressing the signal from the ceiling and/or the floor.

Figure 12 shows the image produced using signals that come from angles greater than $30^{\circ}$ from the vertical direction. Figure 13 shows the image produced using signals that come from angles less than $-30^{\circ}$ from the vertical direction. Compared with the real environment, it was confirmed that white dots at around $(-200,1,400)$ in Fig. 12 were signals reflected from the ceiling and the responses in Fig. 13 were from the floor and the aliasing image of the ceiling.

Using an M-sequence signal and the arrival direction

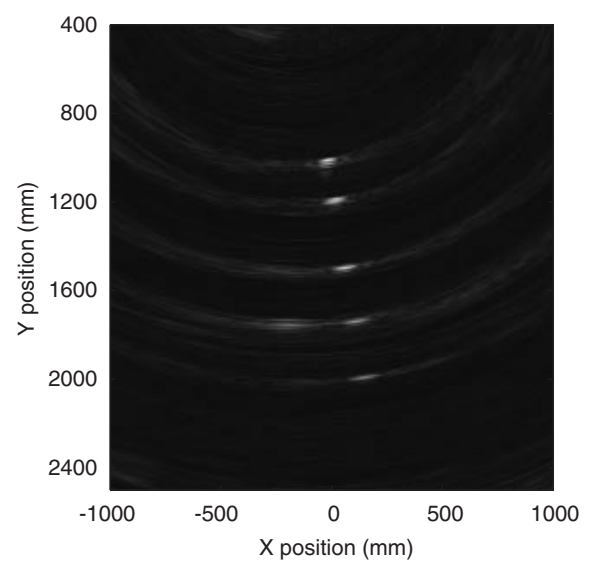

Fig. 11 Image of 2-dimensional plane in the horizontal direction $\left(-30^{\circ} \leq \theta \leq 30^{\circ}\right)$.

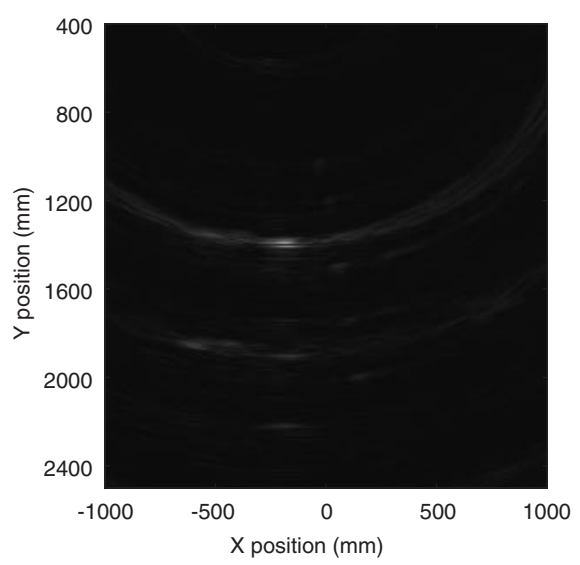

Fig. 12 Image formed by using upward direction information $\left(30^{\circ}<\theta\right)$.

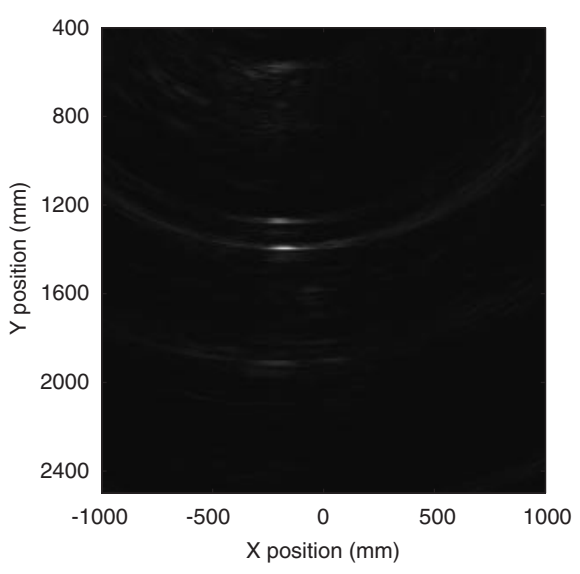

Fig. 13 Image formed by using downward direction information $\left(\theta<-30^{\circ}\right)$.

information, the position detection of small objects in an indoor environment was realized.

\section{CONCLUSIONS}

In this study, we presented results on the position detection of small objects of size comparable to the signal wavelength using an $\mathrm{M}$-sequence signal and the phase information of the received signals in a noisy indoor environment. Using the M-sequence signal, we were able to measure in a stable manner the reflected waves that could not be detected by using a conventional impulsive signal. We were able to acquire the arrival direction of sound in the vertical direction precisely by using the phase information measured using multiple microphones. Small targets were successfully detected in the image, which was generated from the extracted signals without unwanted signals.

\section{REFERENCES}

[1] K. Mizutani, S. Kawabe, I. Saito and H. Masuyama, "Measurement of temperature distribution using acoustic reflector array,” Jpn. J. Appl. Phys., 45, 4516-4520 (2006). 
[2] D. D. Sukmana and I. Ihara, "Surface roughness characterization through the use of diffuse component of scattered aircoupled ultrasound," Jpn. J. Appl. Phys., 45, 4534-4540 (2006).

[3] K. Sasaki, M. Nishihira and K. Imano, "Low-frequency aircoupled ultrasound system beyond diffraction limit using pinhole," Jpn. J. Appl. Phys., 45, 4560-4564 (2006).

[4] J. A. Jiménez, M. Mazo, J. Ureña, A. Hernández, F. Álvarez, J. J. García and E. Santiso, "Using PCA in time-of-flight vectors for reflector recognition and 3-D localization," IEEE Trans. Robotics, 21, 909-924 (2005).

[5] T. Tanzawa, N. Kiyoshiro, S. Kotani and H. Mori, "The ultrasonic range finder for outdoor mobile robots," Proc. IEEE/RSJ Int. Conf. Intelligent Robots and Systems, Vol. 3, pp. 368-373 (1995).

[6] K.-W. Jörg and M. Berg, "Mobile robot sonar sensing with pseudo-random codes," Proc. IEEE Int. Conf. Robotics and Automation, pp. 2807-2812 (1998).

[7] S. Shoval and J. Borenstein, "Using coded signals to benefit from ultrasonic sensor crosstalk in mobile robot obstacle avoidance," Proc. IEEE Int. Conf. Robotics and Automation, pp. 2879-2884 (2001).
[8] T. Yata, A. Ohya and S. Yuta, "A fast and accurate reflecting points measurable sonar-ring system," J. Robotics Soc. Jpn., 17(8), pp. 121-130 (1999).

[9] T. Nakamura, T. Kanaizumi, H. Fujimori, I. Nakano and K. Metzger, "Simultaneous transmission of tomography transceivers using multiple M-sequence signals," Jpn. J. Appl. Phys., 39, 3193-3194 (2000).

[10] Y. Wang, H. Hachiya, T. Nakamura and H. Fujimori, "Estimation of correlation of acoustic reciprocal transmissions using central equatorial pacific tomography data," Jpn. J. Appl. Phys., 44, 4729-4733 (2005).

[11] K. Nishihara, T. Yamaguchi and H. Hachiya, "Precise position measurement of objects using a coded acoustic signal in air," Proc. Autumn Meet. Acoustic. Soc. Jpn., pp. 1013-1014 (2006).

[12] M. Okujima, N. Endoh and T. Murotani, "Acoustic imaging using impulsive sound," J. Acoust. Soc. Jpn. (J), 33, 515-523 (1977).

[13] Y. Wang, H. Hachiya, T. Nakamura and H. Fujimori, "Precise measurement of travel time difference for acoustic reciprocal transmission," Jpn. J. Appl. Phys., 42, 3206-3211 (2003). 